\title{
NUMERICAL STUDY OF THREE-DIMENSIONAL MODULATIONS IN A SHOCK-INDUCED SEPARATION
}

\author{
L. Agostini ${ }^{1}$, L. Larchevêque ${ }^{1}$, E. Garnier ${ }^{2}$, \\ and E. De Martel ${ }^{2}$ \\ ${ }^{1}$ IUSTI, UMR CNRS 6595 \\ Université Aix-Marseille I \\ Rue Enrico Fermi 5, 13453 Marseille cedex 13, France \\ ${ }^{2}$ ONERA, Applied Aerodynamics Department \\ Rue des Vertugadins 8, Meudon 92190, France
}

Large-eddy simulations (LES) of a shock impinging on a turbulent boundary layer are carried out and they demonstrate good agreement with the experiments. A special emphasis is put on the analysis of the three-dimensional (3D) modulations of the flow in order to clarify the origin of the mean vortices located in the separation region highlighted by the experiment.

\section{INTRODUCTION}

Shock waves impinging on a turbulent boundary layer, if strong enough, result in a separated region located between the feet of the incident and reflected shocks. Such flows exhibit low frequency unsteadiness of both the reflected shock and the separated region. Moreover, recent experiments carried out at IUSTI [1] have highlighted strong 3D modulations of the mean separated flow due to two large counter-rotating vortices. It is unclear if these modulations are fully induced by the separated boundary layer developing on the lateral walls of the wind tunnel or if a transverse 3D instability is amplified due to the lateral confinement by the walls. The present work aims at clarifying that point by means of LES, taking advantage of the easiness in modifying lateral boundary conditions in numerical simulations.

Large-eddy simulation is well-suited to predict the mean flowfield of an impinging shock-turbulent boundary layer interaction, as demonstrated in [2]. Computations relying on such a turbulent modeling are also able to capture the

This is an Open Access article distributed under the terms of the Creative Commons Attribution-Noncommercial License 3.0, which permits unrestricted use, distribution, and reproduction in any noncommercial medium, provided the original work is properly cited. 


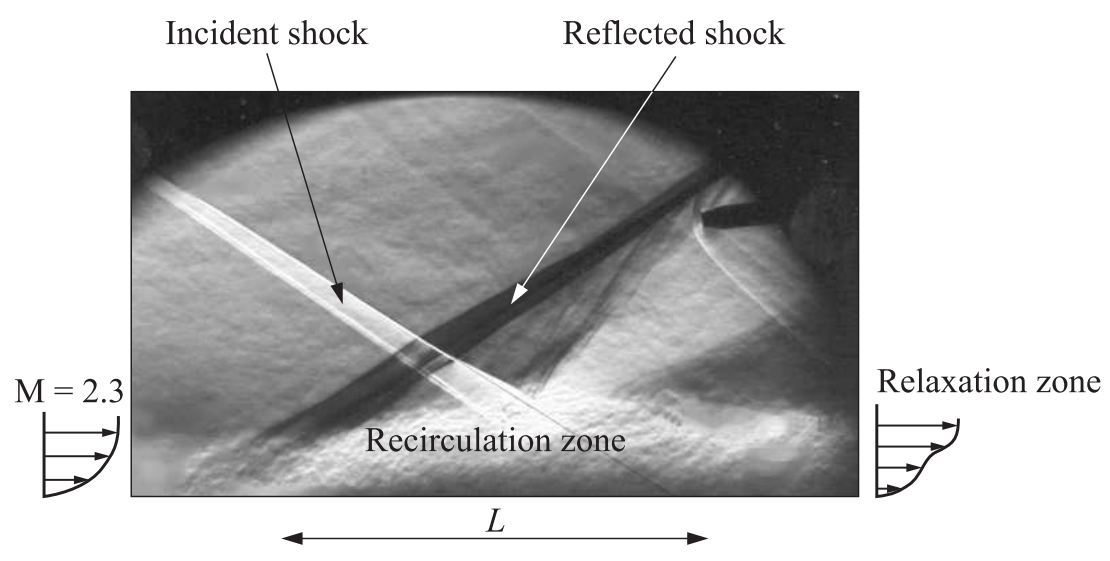

Figure 1 Schlieren picture of the shock-boundary layer interaction

low-frequency unsteadiness of the flow [3]. It is, therefore, expected that LES computations will also be able to reproduce the 3D structures located within the separation as found in the experiments. Four computations have been designed in an attempt to elucidate the origin of the three-dimensionality. They aim at reproducing the experimental case demonstrating the strongest threedimensionality inside the recirculation, which corresponds to a 9.5 degree angle for the shock generator. The incoming flow has a Mach number of 2.3 whereas the Reynolds number of the boundary layer, based on the compressible momentum thickness, is roughly equal to 5000 . The experimental setup can be seen in Fig. 1.

The first and second computations rely on periodicity in the transverse direction, with a width of the computational domain corresponding, respectively, to one tenth and seven tenths of the total width of the wind tunnel. The third and fourth computations include lateral solid walls, either with slip or no-slip conditions. At the time this paper was written, only data from computation 1 (small width with periodicity) and 3 (full width with slip walls) were available and will, therefore, be analyzed hereafter. The second computation (large width with periodicity) has been completed but has not yet been processed.

\section{NUMERICAL METHOD AND COMPUTATIONAL SETUP}

The computation was carried out using ONERA's finite volume FLU3M code (see, for instance, [3] for details). The code is second-order accurate both in 
time and space. Time integration relies on an implicit Gear scheme. The space discretization switches continuously from a centered scheme to a Roe one in the vicinity of the shock according to the Ducros sensor. Turbulent inlet condition is provided by a variant version of the synthetic eddy method [4], fed with velocity profiles coming from the experiments. The inlet is located about 10 boundary layer thicknesses upstream of the shock, ensuring realistic turbulent profiles in the vicinity of the separation.

Either one tenth $(1.7 \mathrm{~cm})$ or the full $(17 \mathrm{~cm})$ width of the wind tunnel are taken into account in the computational domain. The grid resolution is conforming to the LES standards for wall bounded flows with $x^{+} \simeq 45$ (streamwise direction), $y^{+} \simeq 15$ (spanwise direction), and $z^{+}<1$ (wall-normal direction), resulting in 5.5 and 55 million cells meshes. The timestep is set to $2.5 \cdot 10^{-7} \mathrm{~s}$., yielding maximal CFL (Courant-Friedrichs-Levy) values of 12 . Once the initial transient flow has been removed, the computation has been run for either $500 \cdot 10^{-3}$ or $37.5 \cdot 10^{-3} \mathrm{~s}$, corresponding, respectively, to about 130 and 8 periods of the low-frequency unsteadiness of the flow. Unsteady data over various vertical and horizontal planes have been stored with a 200-kilohertz sampling rate. For the full-width computation, 3D short-time averaged data have been saved roughly every one tenth of a period.

\section{VALIDATION}

The two computations yield roughly the same dimension for the separated region, with an average interaction length $L=57 \mathrm{~mm}$ (see Fig. 1) and a separation height of $5.2 \mathrm{~mm}$. These values are respectively underestimated by $20 \%$ and $40 \%$ with regard to the experimental measurements for the 9.5 degree shock generator angle. It is worth noting that similar underpredictions have been found for the 8 degree case by contributors to the European project UFAST [3, 5], as well as for a 5.5 degree computation carried out at IUSTI. The origin of such an underestimation is not yet fully understood but could be due to the curvature of the shock in the spanwise direction induced by the lateral boundary layer found in the experiments [6]. The ongoing fourth computation, taking into account these lateral boundary layers, should help clarifying that point.

The computations can nonetheless be validated by putting aside the shock angle and by focusing on the interaction length. According to this scaling, the 9.5 degree computations match very well the 8.8 degree experiments. A similar concordance is found when looking at the low frequency unsteadiness of the shock. Unfortunately, longitudinal particle image velocimetry (PIV) measurements are not yet available for this angle, but the flow topology that can be inferred from the 8 and 9.5 degree experimental velocity data are in good concordance with the ones found in the computations, as seen in Figs. 2-6. 
Streamwise velocity / $\mathrm{m} / \mathrm{s}$

$0 \quad 100200300400500$

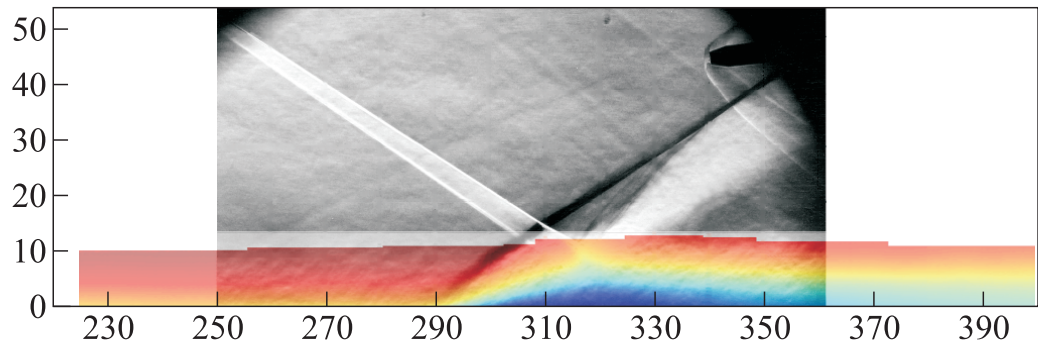

(a)

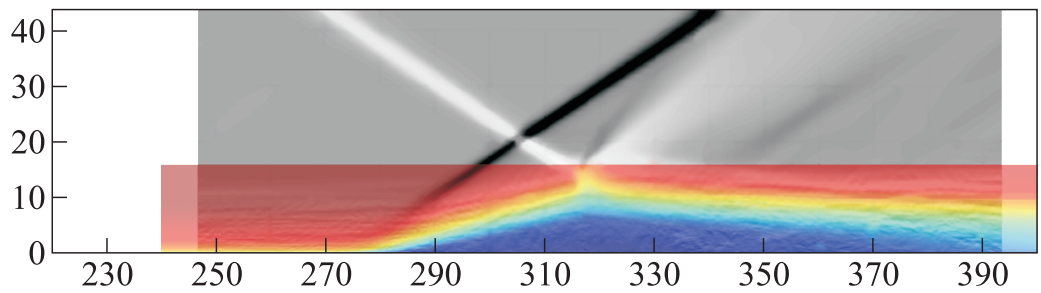

(b)

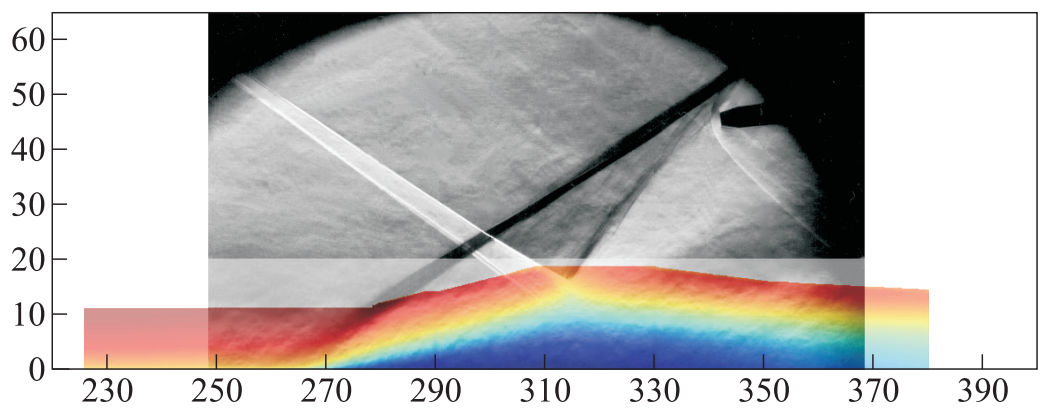

(c)

Figure 2 Superposition of Schlieren views and maps of streamwise velocity from experiments with a 8 degree shock generator $(a)$, LES with a 9.5 degree shock generator $(b)$, and experiments with a 9.5 degree shock generator $(c)$. (Refer Agostini et al., p. 160.) 
Vertical velocity / $\mathrm{m} / \mathrm{s}$

$\begin{array}{llllll}-20 & 0 & 20 & 40 & 60 & 80\end{array}$

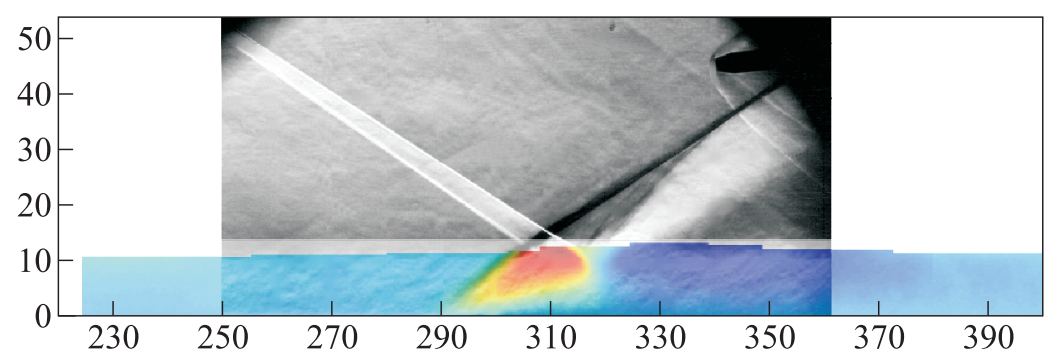

(a)

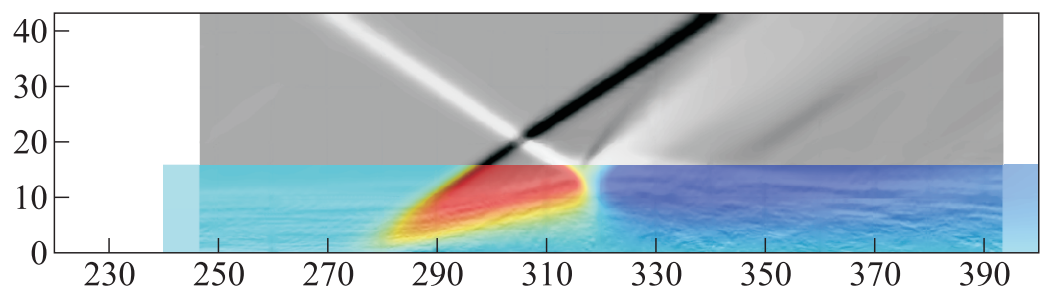

(b)

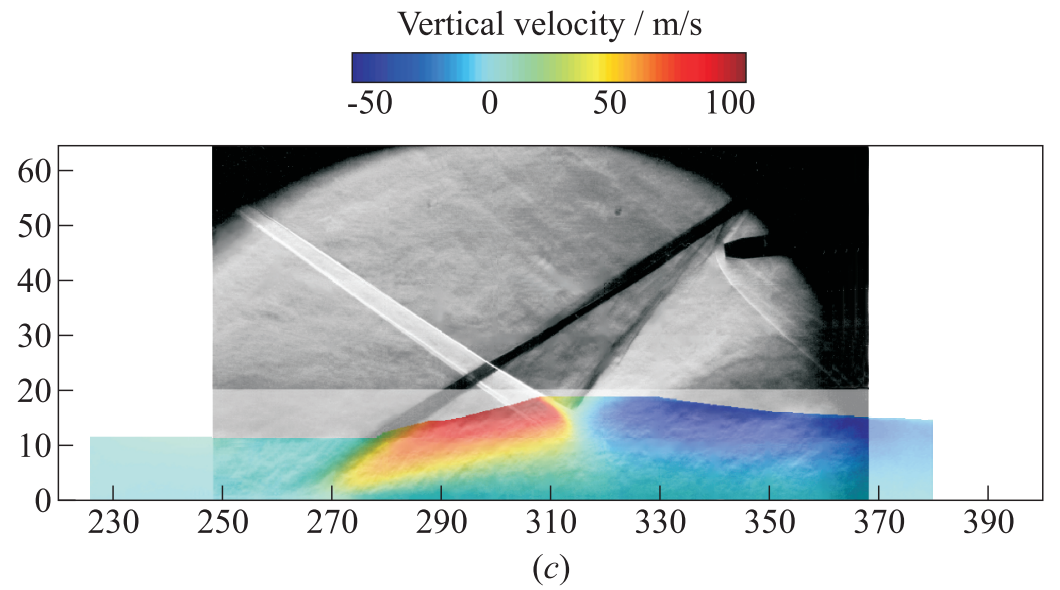

Figure 3 Superposition of Schlieren views and maps vertical velocity from experiments with a 8 degree shock generator $(a)$, LES with a 9.5 degree shock generator $(b)$, and experiments with a 9.5 degree shock generator (c). (Refer Agostini et al., p. 161.) 
Streamwise velocity fluctuations / $\mathrm{m} / \mathrm{s}$

$\begin{array}{llllll}20 & 40 & 60 & 80 & 100 & 120\end{array}$

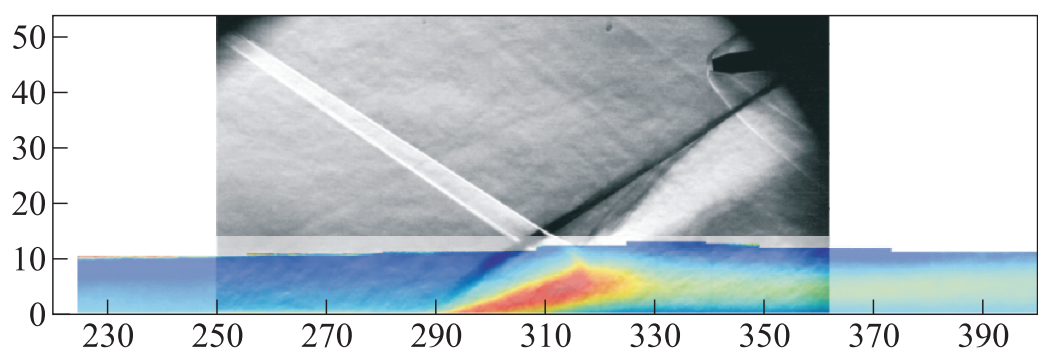

(a)

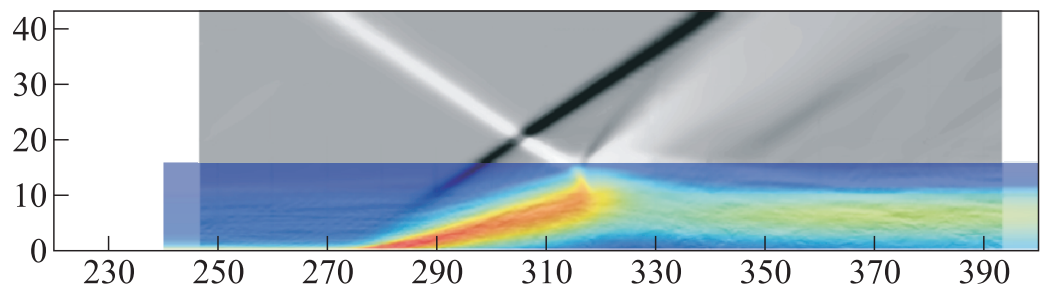

(b)

Streamwise velocity fluctuations / $\mathrm{m} / \mathrm{s}$

$$
20 \quad 40 \quad 60 \quad 80100120140
$$

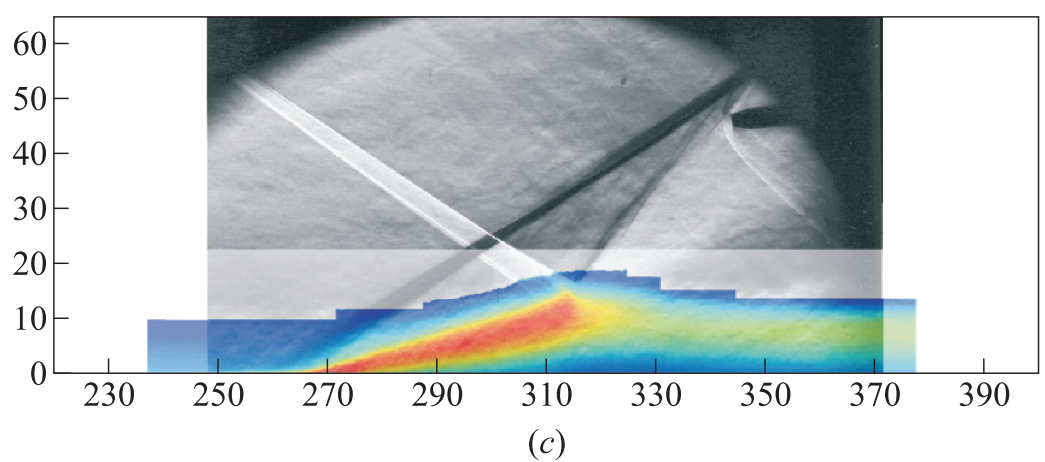

Figure 4 Superposition of Schlieren views and maps of streamwise velocity fluctuations from experiments with a 8 degree shock generator $(a)$, LES with a 9.5 degree shock generator $(b)$, and experiments with a 9.5 degree shock generator $(c)$. (Refer Agostini et al., p. 162.) 


\section{Vertical velocity fluctuations / $\mathrm{m} / \mathrm{s}$}

$\begin{array}{lllllll}10 & 15 & 20 & 25 & 30 & 35 & 40\end{array}$

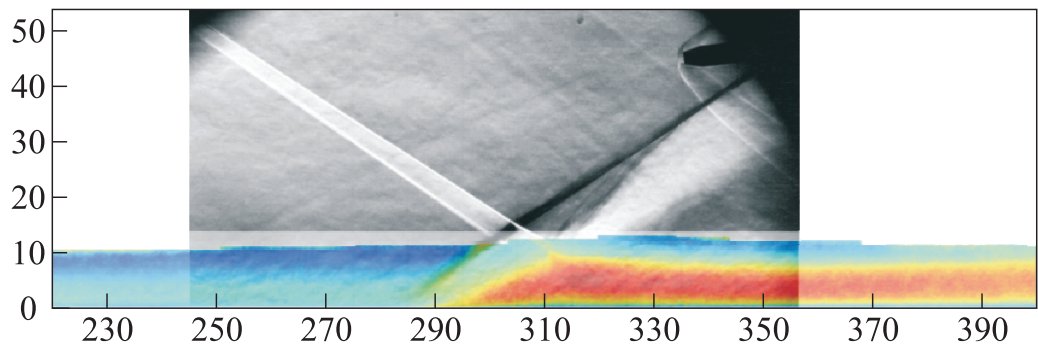

(a)

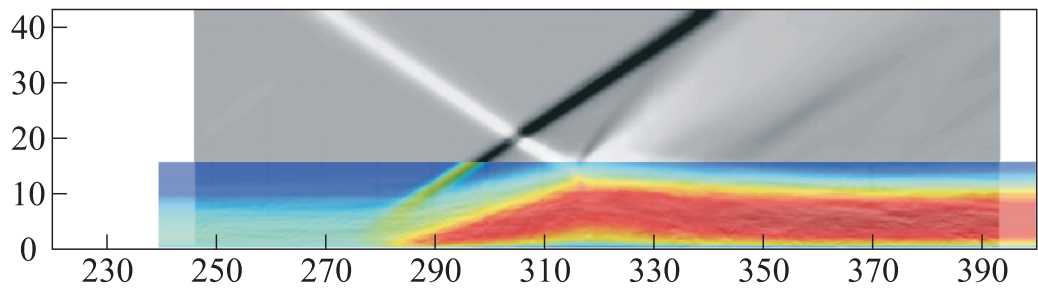

(b)

Vertical velocity fluctuations / $\mathrm{m} / \mathrm{s}$
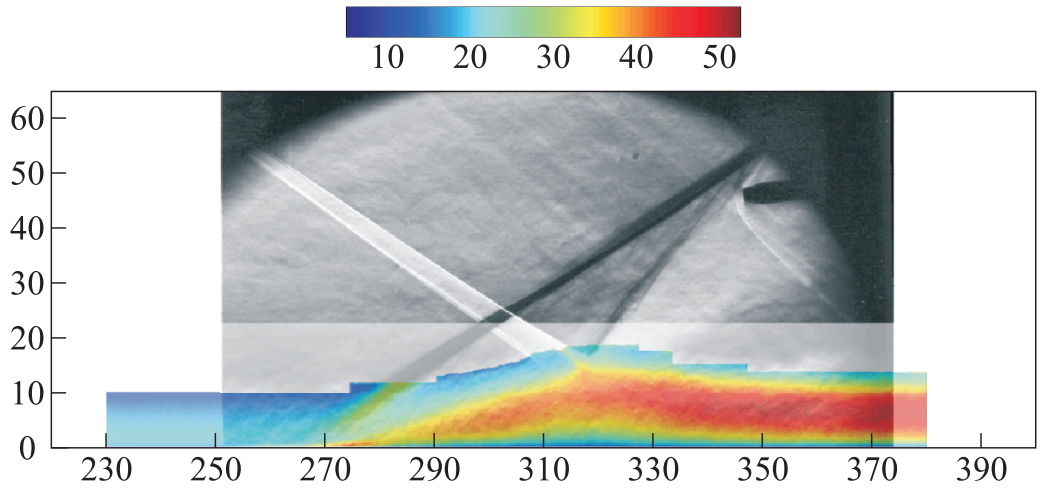

(c)

Figure 5 Superposition of Schlieren views and maps of vertical velocity fluctuations from experiments with a 8 degree shock generator $(a)$, LES with a 9.5 degree shock generator $(b)$, and experiments with a 9.5 degree shock generator $(c)$. Refer Agostini et al., p. 163.) 
Cross streamwise-vertical velocity fluctuations $/ \mathrm{m} / \mathrm{s}$

$$
-1000-500 \quad 0 \quad 50010001500
$$

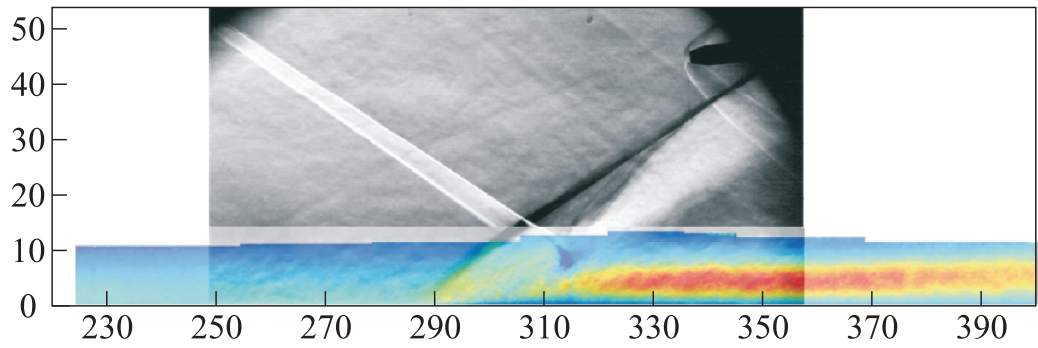

(a)

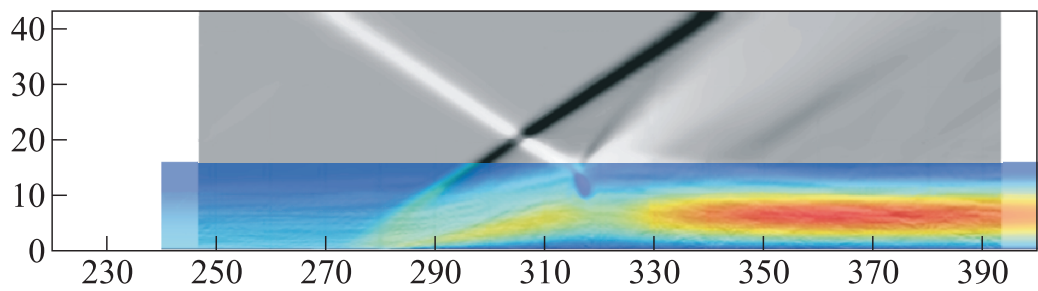

(b)

Cross streamwise-vertical velocity fluctuations $/ \mathrm{m} / \mathrm{s}$
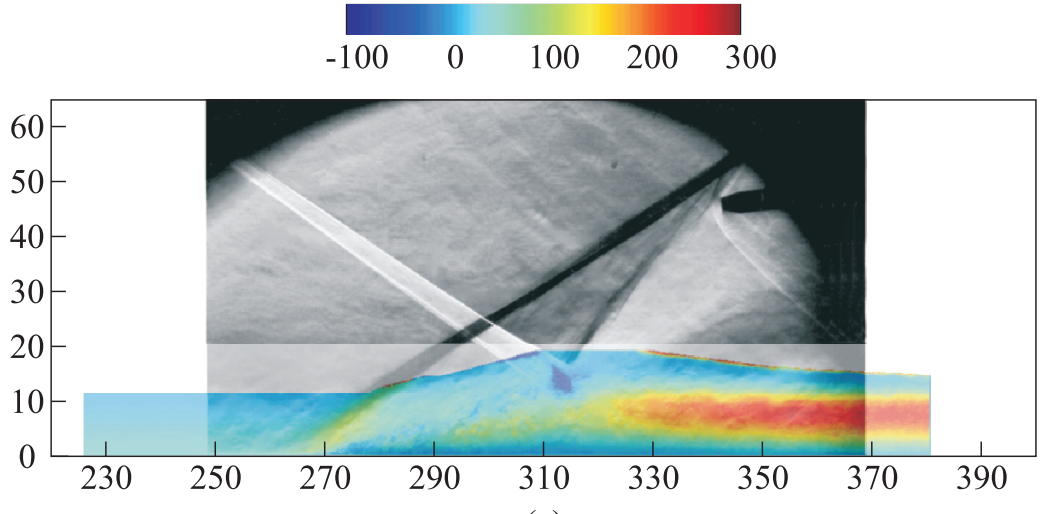

(c)

Figure 6 Superposition of Schlieren views and maps of cross streamwise-vertical velocity fluctuations from experiments with a 8 degree shock generator $(a)$, LES with a 9.5 degree shock generator $(b)$, and experiments with a 9.5 degree shock generator $(c)$. (Refer Agostini et al., p. 164.) 
Consequently, it is assumed that the present computations are able to fully reproduce the physics of the shock - boundary layer interaction highlighted by the experiments despite a lower sensitivity of the separation to the shock angle.

\section{RESULTS}

When considering the full-width computation, persistent spanwise modulations of the velocity flowfield are clearly found in the separated region, as seen in Fig. $7 a$ where the isosurface of null longitudinal mass flux is plotted to visualize the extension of the recirculating flow. Apart of the unphysical increase in the separation size induced by the shock in the vicinity of the slip walls, two regions exhibiting a higher extension of the separated zone are found on both parts of the longitudinal symmetry plane. The distance between these two regions is equal to about $30 \%$ of the total width and is similar to the one extracted from horizontal PIV planes for a shock generator angle of $8.8^{\circ}$. It is worth noting that the span average of the flowfield, excluding the regions close to the wall, results in a height of the separated region almost identical to the one extracted from the small-width computation with periodical boundary conditions.

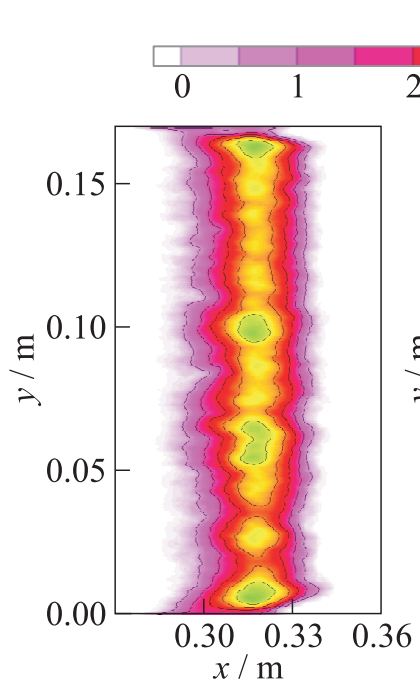

(a) $z / \mathrm{m}$

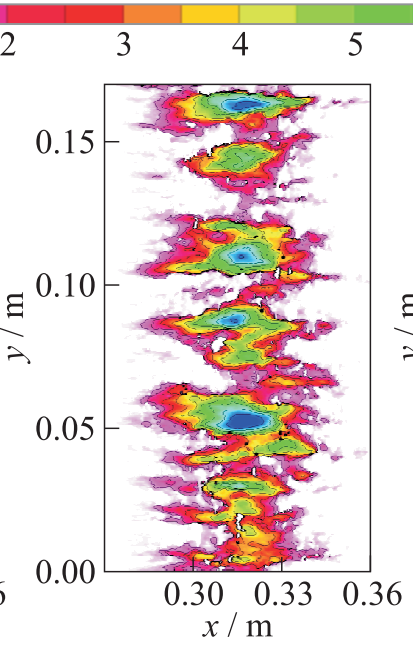

(b)

Figure 7 Elevation of the null longitudinal mass flux surfaces: full-time average $(a)$ and two samples of short-time averages (one tenth of a period) (b). (Refer Agostini et al., p. 165.) 


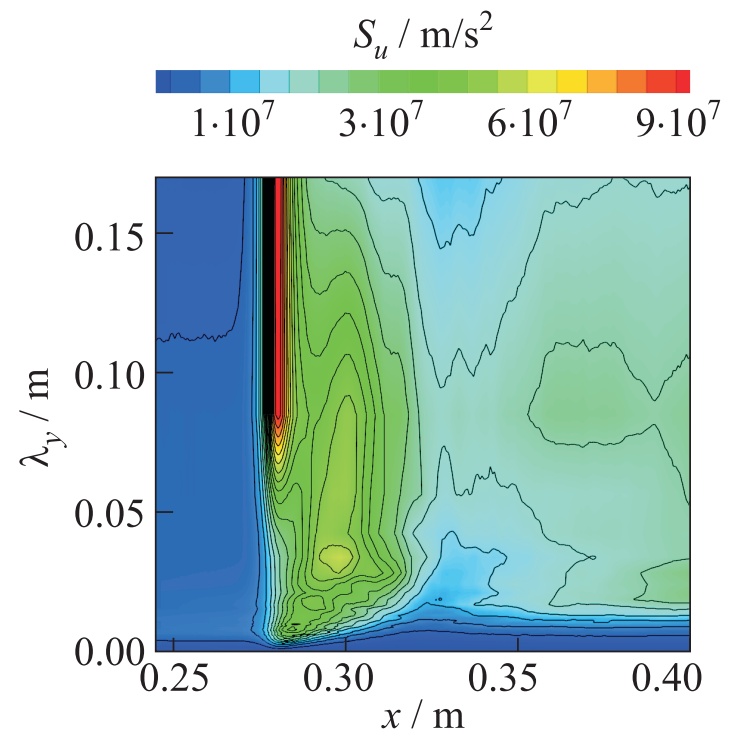

(a)

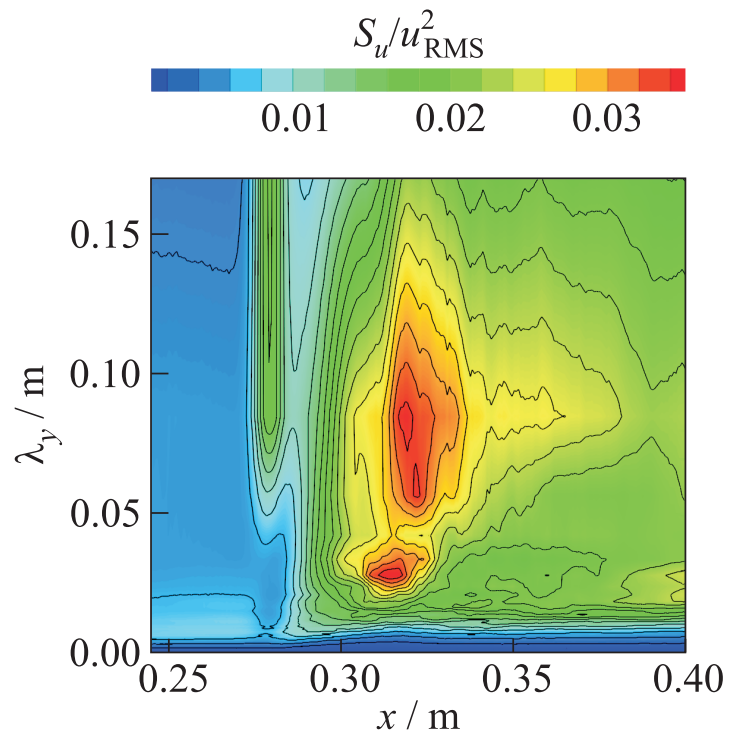

(b)

Figure 8 Longitudinal evolution of the spanwise power spectra of velocity for $z$ $=1 \mathrm{~mm}$ : unormalized spectrum $(a)$ and spectra normalized by the local longitudinal RMS value (b). (Refer Agostini et al., p. 166.) 
Stronger structures are found when looking at short-time averaged flows. The average time considered here corresponds to about 50 characteristic time of the coherent structures located inside the boundary layer and to 0.1 characteristic time of the low-frequency modulation. Therefore, the structures highlighted in Fig. $7 b$ are related to intermediate frequencies similar in magnitude to the typical frequencies of the mixing layer developing over the separated region. It is seen from Fig. 7 that such structures exhibit a maximum height twice larger than the one found on average and are separated by channels of flows with strictly positive local longitudinal mass fluxes. The spanwise separation distance between structures is two to three time smaller than the distance between the two central structures extracted from the mean flowfield.

Spanwise Fourier transforms are used to better characterize the typical lengthscale of the structure. When looking at the unnormalized power spectra of velocity plotted in Fig. 8a, spanwise modulations with largest amplitude are found near the foot of the reflected shock $(X \simeq 0.28)$, with wavelength higher than half the width of the wind tunnel. Milder modulations of lower wavelength are found in the beginning of the separated region $(0.28<X<0.3)$. However, it is worth noting that these two regions roughly match regions with the highest turbulent kinetic energy content.

Consequently, a less biased measure of the 3D nature of the flow can be obtained by locally normalizing the power spectrum using the spanwise root mean square (RMS) value, resulting in Fig. $8 b$. It is seen from this plot that the strongest relative $3 \mathrm{D}$ modulations are located at the core of the separated region $\left(X \simeq 0.32\right.$ ) with typical transverse wavelengths $L_{y}$ of $0.03,0.06$, and $0.085 \mathrm{~m}$. The first value corresponds to the structures seen in Fig. $7 b$ whereas the two last one are more likely related to the structures found in Fig. $7 a$. Interestingly, the $L_{y} \simeq 0.03 \mathrm{~m}$ structures have been found in other shock-boundary layer computations [5] with almost no mean spanwise modulation and bear resemblance with transverse modulations found in other separated flows and related to centrifugal instabilities.

\section{CONCLUDING REMARKS AND FUTURE WORKS}

Various unsteady spanwise modulations have been identified in a shockboundary layer interaction. The ones exhibiting the smallest wavelength seem to be intrinsic to the separated region, may be related to centrifugal instabilities but appear not to affect the mean flow. On the contrary, traces of the modulations with larger wavelengths are found in the averaged flowfield, although they do not affect the global physics of the interaction that remains identical to the one highlighted by the computation of small span, where such modulations cannot be sustained. It is, however, unclear if the lateral walls are responsible for these modulations. 


\section{ACKNOWLEDGMENTS}

This work was partly supported by the CNES through a grant of the pôle ATAC. Computational resources were provided by the IDRIS supercomputer center of the CNRS under project No. 91877 .

\section{REFERENCES}

1. Dupont, P., C. Haddad, J.P. Ardissone, and J.-F. Debiève. 2005. Space and time organisation of a shock wave/turbulent boundary layer interaction. Aerospace Sci. Technol. 9(7):561-72.

2. Garnier, E., P. Sagaut, and M. Deville. 2002. Large eddy simulation of shock/boundary layer interaction. AIAA J. 40(10):1935-44.

3. De Martel, E., E. Garnier, and P. Sagaut. 2007. Large eddy simulation of impinging shock wave/turbulent boundary layer interaction at $\mathrm{M}=2.3$. IUTAM Symposium on Unsteady Separated Flows and their Control. Corfu, Greece.

4. Jarrin, N., S. Benhamadouche, D. Laurence, and R. Prosse. 2006. A synthetic-eddymethod for generating inflow conditions for large-eddy simulations. Int. J. Heat Fluid Flow 27(4):585-93.

5. Touber, E., and N.D. Sandham. 2008. Oblique shock impinging on a turbulent boundary layer: Low-frequency mechanisms. 38th AIAA Fluid Dynamics Conference. Seattle, U.S.

6. Garnier, E. Private communication. 